\title{
Damage Survivability of Cruise Ships - Evidence and Conjecture
}

\author{
Dracos Vassalos, The Ship Stability Research Centre, Department of Naval Architecture, Ocean \\ and Marine Engineering, University of Strathclyde, Glasgow, Scotland, UK, \\ d.vassalos@strath.ac.uk
}

\section{ABSTRACT}

This paper delves into damage stability legislation as it applies to passenger ships. The Concordia accident, like many others before it, has shaken the maritime profession once again with many questions being asked without being able to provide credible answers. Old ships have been designed to lower standards (it is common knowledge that new ships are safer than old ships, with the latter comprising the majority of the population), new standards are holistic and goal-based offering knowledge of the standard these ships are designed to, which is not true for old ships, emergency response is an altogether different science in modern ships and many others. Notwithstanding this state of affairs, there is another more fundamental weakness in the regulations for damage stability, perhaps at the heart of most problems with cruise ships safety, old and new. A critical review into damage stability legislation, as it applies to passenger ships, offers compelling evidence that cruise ship characteristics and behaviour have not been accounted for in the derivation of relevant damage stability rules. As a result, the regulatory instruments for damage stability currently in place do not provide the right measure of damage stability for cruise ships and, even more worryingly, the right guidance for design improvement. This leads to a precarious situation where cruise ships are underrated when it comes to assigning a damage stability standard whilst depriving designers of appropriate legislative instruments to nurture continuous improvement. Documented evidence is being presented and the ensuing results and impact discussed. Recommendations are given for a way forward.

Keywords: damage stability and survivability, cruise ships

\section{INTRODUCTION}

SOLAS regulations is the Bible of safety and like the latter, it is considered "holy" by many and it will take endless debates to change a line, even though the former has been written, in the best of circumstances, by naval architects not yet canonised. A passenger ship is a vessel carrying 12 or more passengers (... and is involved in international trade), irrespective of size, shape, age, construction and condition. This state of affairs has served the maritime industry well for over a century, as it has taken half as long for all concerned to realise that current rules are becoming progressively less relevant and amendments have run their course. The Secretary General of the International Maritime Organization (IMO) Koji Sekimizu, realising fully this state of affairs has set 2029 (the 100th anniversary of SOLAS) as the date by which a new, more relevant, SOLAS will be introduced. Sadly, he is leaving in less than a year's time and the chance that another Naval Architect will be filling his shoes is slim. In the interim, we have reached the embarrassing situation of having to conceal knowledge on the fact that treating all IMO-defined passengers ships the same, is alienating the 
profession when it comes to developing and setting standards for damage stability. It is certain there are many other "anomalies" in SOLAS concerning all sort of different issues but damage stability is big enough a subject when it comes to passenger ships to consider it in isolation. More specifically, there is documented evidence to demonstrate that passenger ship damage stability rule development to date is based almost $100 \%$ on cargo ships and, more recently, on RoRo passenger vessels (Project HARDER, 2003). Whilst the difference between cargo ships and RoRo passenger vessels in terms of damage stability and survivability might be obvious, concerning in particular water on car deck (a characteristic vulnerability of RoPax), any such differences between cruise ships and RoPax are not so obvious to non-specialists. A few points worth mentioning here include:

- Whilst the difference between cargo ships and RoRo passenger vessels in terms of damage stability and survivability might be obvious, concerning in particular water on car deck (a characteristic vulnerability of RoPax), any such differences between cruise ships and RoPax are not so obvious to nonspecialists. A few points worth mentioning here include:

- Old cruise ships (generally with small metacentric height) may capsize during the transient phase of flooding as a result of multiple free surfaces (Vassalos, et al, 2006) whilst RoPax may capsize as a result of progressive flooding and water accumulation on the car deck. Having said this, legislation is focusing on new ships, which is also the focus in this paper.

- As such, new Cruise ships, having very large metacentric height and increased internal layout complexity when upper decks are involved in the flooding process, face slow sinking/capsize (Papanikolaou, et al, 2013). RoPax are vulnerable to rapid capsize (NWEP, Svensen and Vassalos).
- Moreover, in many cases, cruise ships capsize or sink following up-flooding. As a result, the time to capsize with cruise ships is becoming even longer; hours (with cruise ships) rather than minutes (with RoPax).

- Accounting for all the above, damage stability/survivability, post-SOLAS 2009, focusses on the whole-ship concept rather than the ship hull (below the subdivision deck, as it is the case with cargo ships). As such and because of the above, geometric modelling for damage stability calculations differs. With RoPax the car deck is considered as part of the survivability studies whilst in cruise ships at least two additional decks are accounted for.

- This being the case, progressive flooding in cruise ships (the cause for eventual sinking and capsize) is very much different due to the complexity of the internal architecture as compared to RoPax, for example. The whole emphasis on damage stability and survivability changes.

- Cruise ship survivability is more affected by details in local geometry as these impact on progressive flooding whilst RoPax are affected by global parameter changes (e.g., beam, freeboard) as the design vulnerability to flooding refers to the car deck.

- As a result of the above, flooding of cruiseships (ships with complex internal subdivision) is inherently uncertain; there are multiple paths to same end state/different end state from similar initial conditions during periods of time that are similarly uncertain.

- In all research on damage stability ad survivability to date, leading to new legislation, very little effort has been expended on cruise ships (one experimental point in Project HARDER and one in Project GOALDS), Figures 3 and 5, respectively. 
- There is, of course, one additional issue; perhaps the most important of all. Cruise ships are knowledge intensive with innovation a primary ingredient for success. As such, they are safety critical, considering the thousands of people onboard some of the modern megaships. Therefore, from a societal risk perspective, cruise ships warrant much more attention than any other ship type when it comes to damage stability and survivability following collision / grounding; a contribution of some $90 \%$ to total risk of passenger ships.

Because of all the above and, in particular, lack of understanding and hence attention on the damage stability of cruise ships, an unfathomable situation has arisen where cruise ship damage stability is underrated by the rules whilst rendering any attempts to improve damage stability of cruise ships futile, using current IMO cost-effectiveness criteria for decision making. This was the overriding conclusion of a recently completed project on the damage stability of passenger ships, where cruise ships and RoPax have been considered (EMSA III Project, 2015).

This is a precarious position for the cruise ship industry to be in for both the safetycultured and the rule-evading owners; the former because the current regulatory framework does not justify improving cruise ship safety, which we know cannot be right, and the latter because newbuildings cruise ships can easily meet the common "passenger ships pool" regulations and are relaxed in this futility. This situation must change. We must change it. As Naval Architects, we owe it to the travelling public, who board these ships by the thousands at a time.

\section{PROBABILISTIC CONCEPT OF SHIP SUBDIVISION}

\subsection{Conceptual Formulation}

A direct link between the probabilistic concept of ship subdivision and modern concepts of risk estimation may simplistically be expressed as follows:

$R_{c}=P_{c} \times P_{w / c} \times P_{f / w / c} \times P_{1 / f / w / c}$

Where:

$\mathrm{R}_{\mathrm{c}}-$ Collision risk

$\mathrm{P}_{\mathrm{c}} \quad$ Probability of a collision event, dependent on loading condition, area of operation, geography, topology, bathymetry, route, traffic density, ship type, human factors, etc.;

$\mathrm{P}_{\mathrm{w} / \mathrm{c}} \quad$ Probability of water ingress, conditional on collision event occurring (accounting for all the above);

$\mathrm{P}_{\mathrm{f} / \mathrm{w} / \mathrm{c}}$ Probability of failure (capsize / sinking / collapse), conditional on collision and water ingress events occurring expressed as a function of e.g., sea state, structural strength and time;

$\mathrm{P}_{1 / f / \mathrm{w} / \mathrm{c}}$ Consequences (Probability of Loss) deriving from the collision event, conditional on all the foregoing; this accounts for loss of (or injury to) life, property damage / loss and impact to the environment. The former will depend on time to capsize and time to abandon ship (as determined from evacuation analysis - passenger ships) and the latter of e.g., probabilistic oil outflow using relevant models of oil spill damages and results from known accidents or through analysis using first-principles tools. 
Considering the above and on the basis of work by (Lutzen, 2001), the relevant probabilities can be calculated from firstprinciples (with appropriate empirical adjustments). Hence, if a more specific analysis is warranted for a novel ship design concept, the probability of collision damage that leads to hull breaching and flooding could be calculated. Moreover, based on work reported in (Jasionowski and Vassalos, 2006) and (Dogliani, et al., 2004), the various terms in [1] could also be addressed for each pertinent scenario from first principles. This allows for complete risk analysis of any damage case.

\subsection{Basic Formulation (SOLAS 2009)}

One of the fundamental assumptions of the probabilistic concept of ship subdivision in SOLAS 2009 is that the ship under consideration is damaged, i.e. the hull is assumed to be breached and there is (large scale) flooding. This implies that the cause of the breach, the collision event and the circumstances leading to its occurrence are disregarded; hence the interest focuses on the conditional probability of survival. Other pertinent factors, such as size of ship, number of persons on board, life-saving appliances arrangement, and so on, are directly or indirectly accounted for by the Required Index of Subdivision R. Therefore, the probability of ship surviving collision damage is given by the Attained Index of Subdivision, A, using the following expressions:

$$
A=\sum_{j=1}^{J} \sum_{i=1}^{I} w_{j} \cdot p_{i} \cdot s_{i}
$$

Where,

$j=\quad$ represents the loading conditions (draught) under consideration
$J=\quad$ is the number of loading conditions considered in the calculation of the attained index (normally 3 draughts)

$w_{j} \quad$ is weighting factor for each draught;

$i$ represents each compartment or group of compartments under consideration for loading condition $\mathrm{j}$

$I$ is the set of all feasible flooding scenarios, comprising single compartments and groups of adjacent compartments for loading condition $\mathrm{j}$; The sum is taken for all cases of flooding in which one, two, three or more adjacent compartments are involved.

$P_{i} \quad$ is the probability that, for loading condition $\mathrm{j}$, only the compartment(s) under consideration are flooded weighted by the probability that the space above a horizontal subdivision may not be flooded (note that $\sum p_{i}=1$ for each draught considered)

$s_{i} \quad$ is the (conditional) probability of surviving the flooding of compartment(s) under consideration for loading condition $\mathrm{j}$

The summation in equation (2) covers only flooding scenarios for which both $p_{i}$ and $s_{i}$ are positive (i.e., survivable scenarios, which contribute to the summation). In other words, $\mathrm{A}$ is the weighted average "s-factor", with " $p$ factors" being the weights, i.e.:

$$
A=\hat{E}(s) \text { on } I
$$

The Attained Index of Subdivision, A, must be greater than the Required Index, $R$, as specified by the regulations, i.e.:

$$
A>R
$$


Deriving from the above, it is further implied that two different ships achieving the same Attained Index of subdivision are equally safe. The philosophy behind the probabilistic concept is that two different ships with the same index of subdivision have equal overall capacity to resist flooding following collision, although these ships may have quite different actual capabilities to withstanding individual damage scenarios (local) in addition to being subjected to different collision risk altogether. Therefore, it is this summary statistic that is the key.

Having said this, there is a profound knowledge hidden in the basic formulation of the probabilistic rules for damage stability, especially when the targeted population is cruise ships, carrying thousands of people onboard. In this case, given that capsize or sinking of any such ship will be catastrophic, the emphasis in the risk model shifts towards damage limitation rather than reducing the probability of such an event taking place. Hence, the emphasis by (Wendel, 1968) on Index-A alone. This is key to understanding Wendel's formulation and to ensuring that no effort will be spared with e.g., large cruise ships to making A as close to 1 as possible.

Considering (1) and (3) and allowing for large time intervals, it is apparent that

$$
\mathrm{P}_{\mathrm{f} / \mathrm{w} / \mathrm{c}}=(1-\mathrm{A})
$$

This means that Index A is the marginal probability for time to capsize within certain time, assuming that the time being considered is sufficiently long for capsize to have occurred in the majority of cases. This is a key observation, as this can be used to derive the flooding risk contribution, as indicated in the following. However, the assumption on time being sufficiently long is critical.

Finally, the Required Index of Subdivision, $\mathrm{R}$ represents the "level of safety" associated with collision and flooding events that is deemed to be acceptable by society, in the sense that it is derived using ships that society considers fit for purpose, since they are in daily operation.

\section{STATUTORY A-INDEX \\ CALCULATION (SOLAS 2009)}

\subsection{Capsize band}

Capsize band is a concept describing the transition of sea-states from those at which no capsize is observed (lower boundary) to those at which the probability of capsize equals unity (upper boundary). In simpler terms, it is a band outside which capsize is either unlikely to happen or certain. For a finite observation time, the probability of capsize can be approximated either as a sigmoid function (Tsakalakis et al, 2010) or alternatively as a Gaussian distribution (Jasionowski et al, 2007). Significantly, it can be observed that as the time of observation increases the capsize band contracts towards its lower boundary, becoming a unit step function as time approaches infinity (Figure 1). This property is of major importance, particularly when the focus is cruise ships where the time it takes the vessel to capsize is normally much longer than the current SOLAS-based evaluation of 30 minutes. In this respect, Hscrit, is associated with the sea state at which the probability of capsize $\left(\mathrm{P}_{\mathrm{f}}\right)$ is equal to 0.5 , based on 30-minute tests.

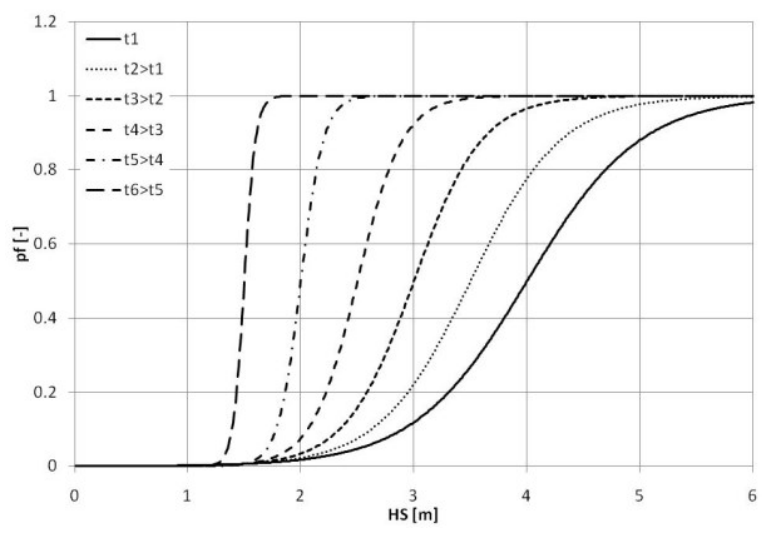

Figure 1: Capsize band as function of the 
observation time, (Tsakalakis, 2012).

\subsection{Survival Factor-s (Projects HARDER and GOALDS)}

Although it is not explicitly stated in SOLAS, the s-factor is a measure of the probability of survival of a damaged ship in waves, namely:

$$
S=\int_{0}^{\infty} d H_{S} \cdot f_{H_{S} \mid c o l l}\left(H_{S}\right) \cdot F_{\text {surv }}\left(H_{S}\right)
$$

Where: $f_{H_{S} \mid c o l l}\left(H_{S}\right)$ is the probability density distribution of sea states expected to be encountered during collision and $F_{\text {surv }}\left(H_{S}\right)$ is the probability of survival in that sea state when exposed to a specific flooding case. More importantly, deriving from the observations made in 3.1 above, the probability of survival is in fact a conditional probability:

$F_{\text {surv }}\left(H_{S}\right) \equiv F_{\text {surv }}\left(t=30 \min \mid H_{S}\right)$

This yield:

$$
\begin{aligned}
& s(t=30 \mathrm{~min})= \\
& =\int_{0}^{\infty} d H_{S} \cdot f_{H_{S} \mid \text { coll }}\left(H_{S}\right) \cdot F_{\text {surv }}\left(t=30 \mathrm{~min} \mid H_{S}\right)
\end{aligned}
$$

Furthermore, it has been assumed that the probability of survival, $F_{\text {surv }}\left(H_{S}\right)$ can be approximated by a step function centred on the sea state. That is, the $\mathrm{Hs}_{\text {crit }}$ constitutes the $50^{\text {th }}$ percentile of the significant wave height the vessel, subjected to a particular damage scenario, can survive for 30 minutes (this corresponds to the abscissa of the inflection point of the sigmoid that defines the capsize band, obtained for $\mathrm{t}=30 \mathrm{~min}$ ). In Project GOALDS, the capsize band itself was substituted by a step function, as outlined next:

$$
F_{\text {surv }}\left(H_{S}\right)=\left\{\begin{array}{l}
1 \Leftrightarrow H_{S} \leq H_{\text {Scrit }} \\
0 \Leftrightarrow H_{S}>H_{\text {Scrit }}
\end{array}\right.
$$

On the basis of this, the final formulation becomes:

$$
\begin{aligned}
S & =\int_{0}^{H_{\text {Scrit }}} d H_{S} \cdot f_{H_{S} \mid \text { coll }}\left(H_{S}\right)= \\
& =\exp \left(-\exp \left(0.16-1.2 \cdot H_{\text {Scrit }}\right)\right)
\end{aligned}
$$

Where the $H_{S \text { crit }}$ is given as:

$$
\begin{aligned}
& \left.H_{\text {Scrit }}\right|_{t=30 \min }= \\
& =4\left(\frac{\min \left(G Z_{\max }, 0.12\right)}{0.12} \frac{\min (\text { Range }, 16)}{16}\right)= \\
& =4 \cdot s(t=30 \mathrm{~min})^{4}
\end{aligned}
$$

In essence, the approach adopted within the GOALDS Project is similar to that of the HARDER project with the main difference stemming from the assumption of $H s_{\text {crit }}$ corresponding to the lower limit of the capsize band, thus allowing for a justified assumption of very long ("infinite") time of survival. Therefore, the limiting assumption of short survival time, implicit in the formulation of HARDER has been addressed properly in GOALDS. This makes the GOALDS s-factor formulation better suited to cruise ships than the current SOLAS formulation.

Moreover, in the analysis of results pertaining to small and large vessels (sample ships in Project GOALDS), it was made apparent that there is a significant effect deriving from scale. Indeed, one of the major concerns related to SOLAS 2009 formulation for the s-factor was that it does not account for the ship size and that it might be inaccurate when applied to vessels deviating significantly from the size of the test vessels used in HARDER as basis for its derivation. In addition, the fact that the SOLAS $2009 \mathrm{~s}$-factor formulation (residual GZ curve characteristics) 
is limited to relatively small range and maximum GZ values fails to account for the contribution of watertight volume distributed high enough not to be "seen" by the formulation. This, in essence deviates from normal Naval Architecture practice, previously expressed through the explicit demand for and provision of residual/effective freeboard.

Accounting for the above and using a systematic approach based on applying Design of Experiments (DoE), the formulation finally proposed is given by the following expression (Cichowicz, et al. 2011):

$$
H_{\text {Scrit }}=\frac{A_{G Z E}}{\frac{1}{2} G M_{f} \cdot \text { Range }} V_{R}^{1 / 3}
$$

And,

$s\left(H_{S}\right)= \begin{cases}e^{\left(-e^{\left(0.16-1.2 H_{S c r i t)}\right)}\right)}, & \forall\left(A_{G Z}, V_{R}, \text { Range, } K>0\right)(13) \\ 0, & \text { otherwise }\end{cases}$

Where $A_{G Z E}$ is an effective area under the GZ curve taken up to the heel angle corresponding to the submersion of the opening in question and VR is the residual volume mentioned above; $G M_{f}$ is residual metacentric height. This formulation, by incorporating residual volume accounts for the effect of scale on one hand whist on the other incorporates a key feature of the cruise vessels, namely residual volume high up in the vessel, which is a key characteristic of modern cruise vessel design.

The overall improvement between Projects HARDER (SOLAS 2009) and GOALDS, pertaining to RoPax, is best visualised in Figure 2 next.
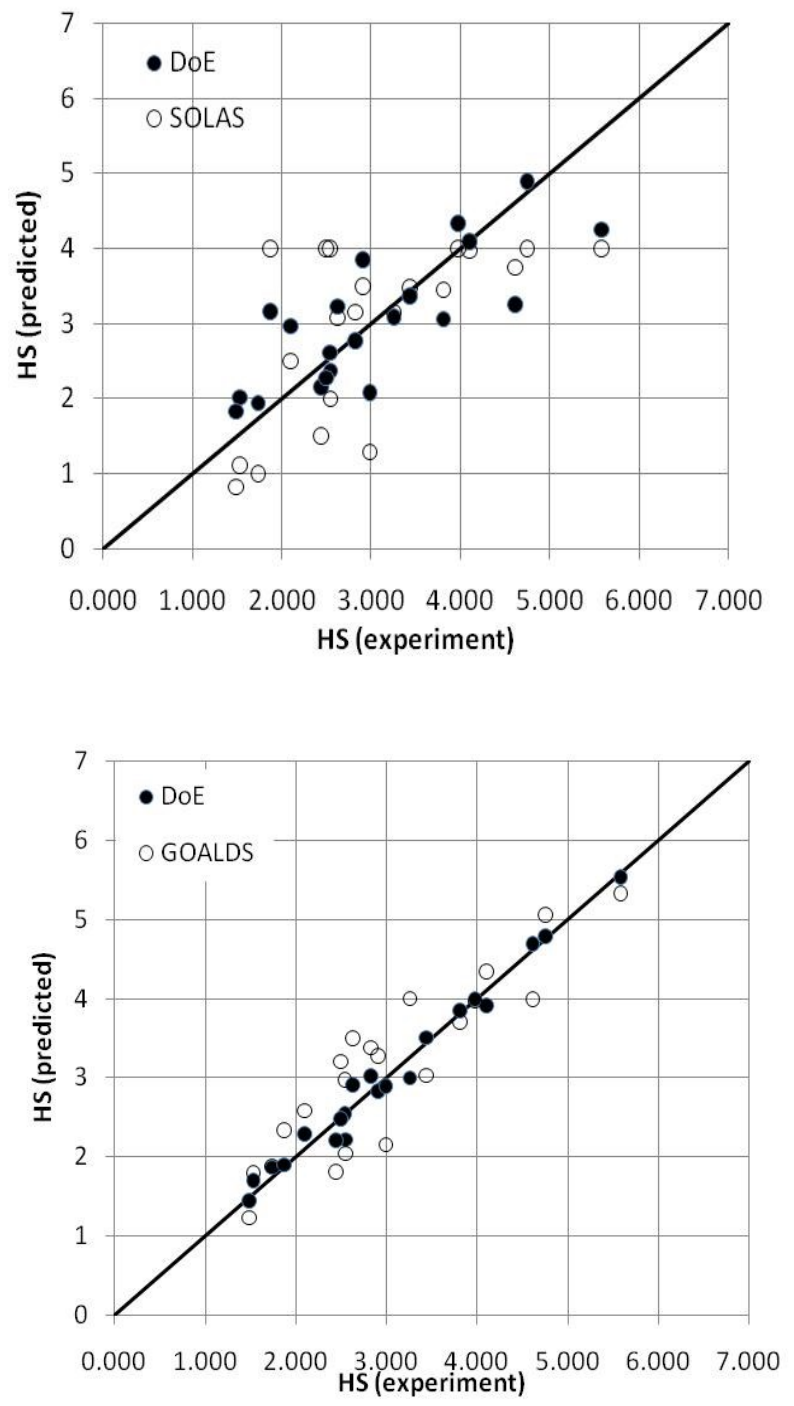

Figure 2: Comparison between predicted and experimental survivability results, using SOLAS 2009 (HARDER - Top) and (GOALDS - bottom) s-factor formulations.

As indicated in the introduction, the formulation for the s-factor in current SOLAS is based almost exclusively on results of either RoPax or cargo ships. The one cruise ship used in GOALDS provides evidence that the SOLAS formulation for s-factors

(a) Does not relate to cruise ships and, this fact leads to another truth, namely that

(b) Current SOLAS does not account for the known survival resilience of cruise ships

Figures 3 and 4 next provide rare evidence. 


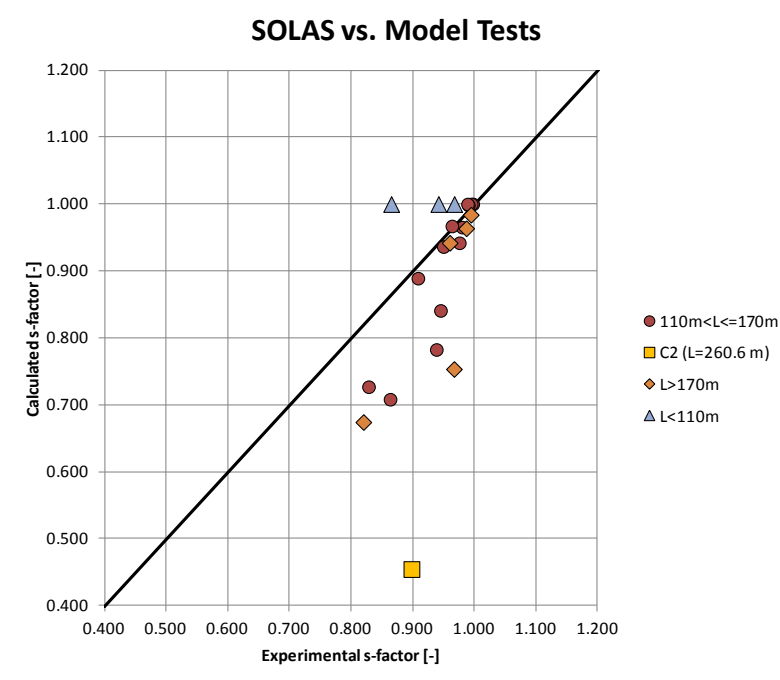

Figure 3: Comparison between predicted and experimental survivability results, using SOLAS 2009 (HARDER) s-factor formulation

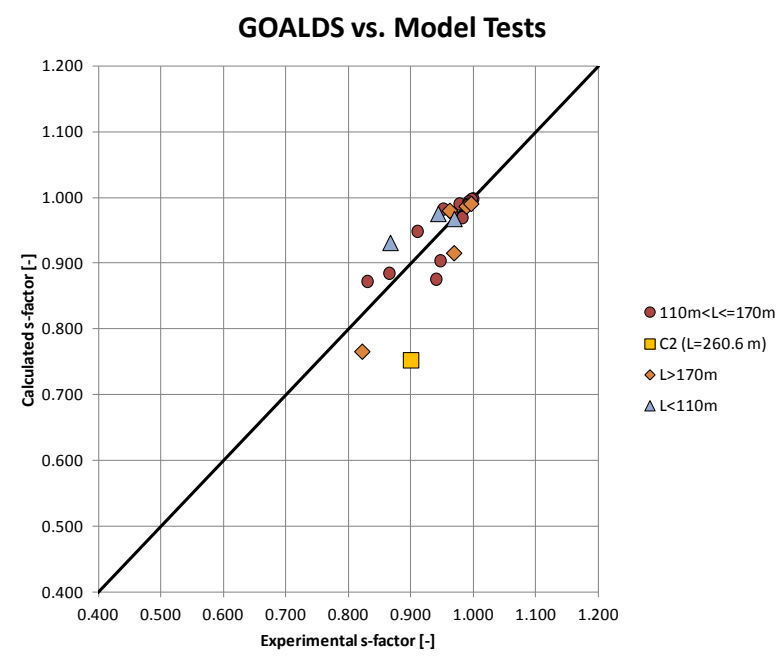

Figure 4: Comparison between predicted and experimental survivability results, using Project GOALDS s-factor formulation

In this light, it is important mentioning here that similar to Project GOALDS, the formulation of the s-factors for the current SOLAS formulation (Project HARDER) contains only one survivability experiment of a cruise ship, which again illustrates higher capsize resilience (Figure 5). The graph also illustrates that the s-factor in current SOLAS is, in fact, based on cargo ships results!

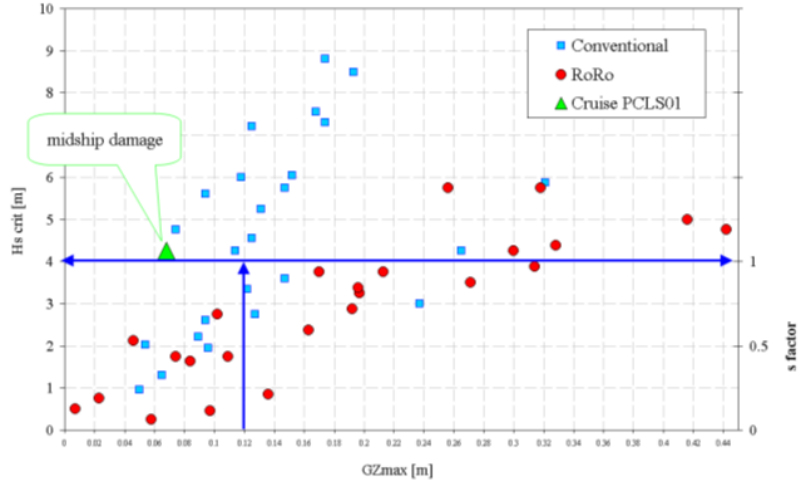

Figure 5: Experimental damage survivability results derived to support SOLAS 2009 (HARDER) s-factor formulation, using cargo ships (conventional), RoRo and 1 cruise ship

\section{DIRECT APPROACH A-INDEX DERIVATION}

\subsection{Approval of Alternative and Equivalents}

With direct influence from regulations, and because of the level of effort that is still needed to implement Risk-Based Design (RBD) in full, the real inno5vation attributable to RBD is currently witnessed mainly at local level. Known as "Approval of Alternatives and Equivalents" (MSC.1/Circ. 1455, 24 June 2013), it is using the principle of equivalent safety to consider alternative design and arrangements other than those supported by SOLAS legislation. This has taken a more generalised character than initially envisaged, with legislative instruments currently in place to address Fire Safety (SOLAS II-2, Reg. 17, MSC/Circ.1002); Life Saving Appliances (SOLAS III/Reg. 38, MSC/Circ. 1238), Damage Stability (Ch. II-1, Re, 4) and general Approval of Equivalents (MSC/Circ. 1455).

This opens the door to using an equivalent approach to A-Index derivation, as reported in (Vassalos et al, 2008) and highlighted in the following. 


\subsection{Impact of Time to Capsize}

As discussed earlier, the survival factor "s" is estimated based on the assumption that the ship capsizes within half an hour, deriving mainly from work on RoPax. This, however, is not the case with cruise ships and it will be of interest to have another introspective look into this with the view to ascertaining the impact of a more prolonged time to capsize. The time to capsize (tc), is a random variable, hence only known as a distribution determined through probability methods. Moreover, it is dependent upon a number of parameters (e.g. flooding condition, sea state, damage extent) all of which are also random in nature. In this respect, accounting only for the damage case scenarios implicit in SOLAS 2009 (normally over 1,000 for a typical passenger ship) and considering the 3 loading conditions, also implicit in the rules, and some 10 sea states per damage case, it becomes readily obvious that some form of simplification and reduction will be meritorious. In view of this, two lines of action have been pursued and two methods are currently available. The first relates to the development of a simple (inference) model for estimating the time to capsize, for any given collision damage scenario; the second entails automation of the process using Monte Carlo sampling of the random variables and time domain simulation, as outlined next.

\section{Method 1: Univariate Geometric Distribution}

Considerable effort has been expended over many years to develop an analytical expression, which could provide an overall description of the character of the stochastic process of ship capsize when subjected to collision damage in a seaway, (Jasionowski, et. al, 2004, 2006, 2008). The inference model used is based on a Univariate Geometric Probability (UGD) density distribution for time to capsize for each flooding scenario, where the only random variable being considered is the survival factor "s" as defined in SOLAS. Hence, the result will be subjected to the same limiting assumptions, inherent in the rules, e.g., applicable to scenarios where the time to capsize is short, more specifically 30 minutes (SAFEDOR Report, 2006). Figure 6 presents a result for a typical ship at scenario level where using this simple inference model, it is possible to predict instantly the likelihood of a vessel to capsize within a given time in any given flooding scenario.

Scenario $=\{$ displ, KG, damage, Hs $\}$

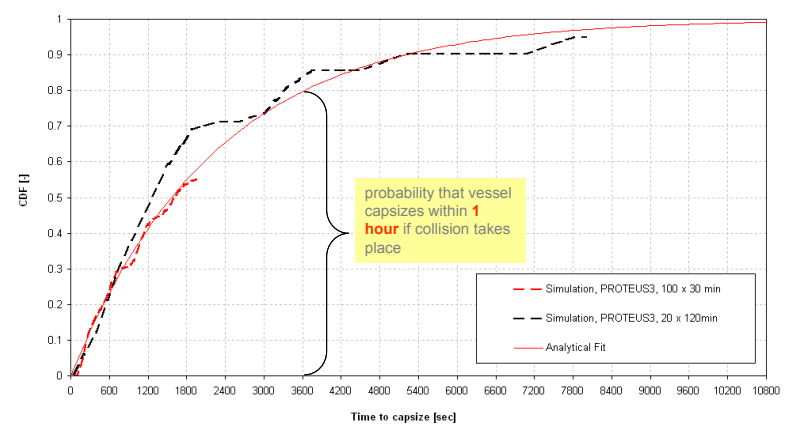

Figure 6: Cumulative probability function for time to capsize (scenario level) - Comparison between analytical model and numerical simulation results

Considering the ease of this operation, tens of thousands of scenarios may be considered to develop pertinent distributions at ship level, see Figure 7. Considering all flooding scenarios of interest for a typical ship, the outcome is the marginal cumulative probability distribution for time to capsize, shown in Figure 7.

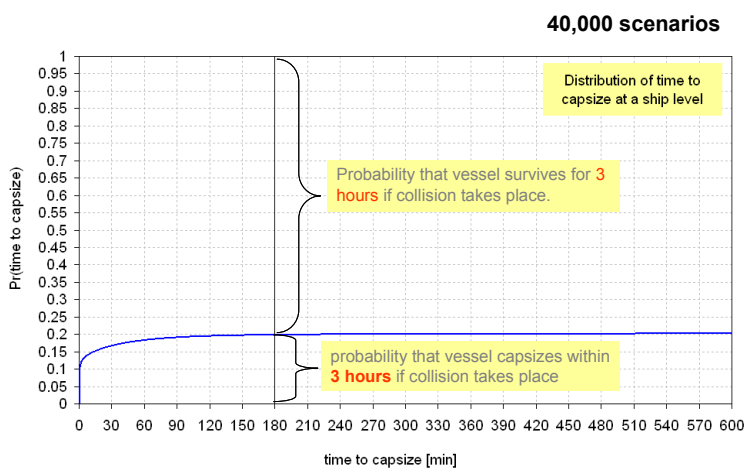

Figure 7: Cumulative marginal probability distribution for time to capsize

A close examination of Figure 7 reveals the following noteworthy points: 
- If a vessel did not capsize within the first hour post-accident, capsize is unlikely, on average.

- The marginal probability distributions for time to capsize tends asymptotically (i.e., after infinite time, in principle) to values defined by (1-A), as indicated earlier.

\section{Method 2 - Monte Carlo Simulation}

To overcome problems associated with "averaging" the following approach may be adopted instead:

- Use of actual statistics (e.g., loading, sea state, damage size, survival time);

- Account properly for physical phenomena of ship motion and floodwater dispersion;

- Disclose ship attitude and behaviour as a function of time (including time to capsize);

- Aiming to avoid any "unnecessary" conservatism and other approximations and potential weaknesses embedded in the formulation of the probabilistic rules (e.g., heel limitations, down flooding points, etc.), the random variables distributions comprising loading conditions, sea states and damage characteristics are sampled using Monte Carlo Sampling and each ensuing damage scenario is simulated using explicit dynamic flooding simulation by PROTEUS3, (Jasionowski, 2005);

- Random variables to be considered would involve for collision: location, length, height, penetration according to the damage statistics adopted in the probabilistic rules and sea state. The resolution could be as high as necessary (every second of each scenario) accounting for transient- cross- and progressiveflooding, impact of multi-free surfaces, watertight and semi-watertight doors (relevant to cruise ships).

Applications of this method indicate that 500 scenarios would result in an absolute sampling error for the cumulative probability of time to capsize in the order of $4 \%-5 \%$.
Examples of Monte Carlo simulations setup are shown in Figures 8-9 for collision. The coloured bars indicate collision damage cases with the position of the bar designating location at the ship, the length, damage size and the colour wave height from $0 \mathrm{~m} \rightarrow$ green to $4 \mathrm{~m} \rightarrow$ red.

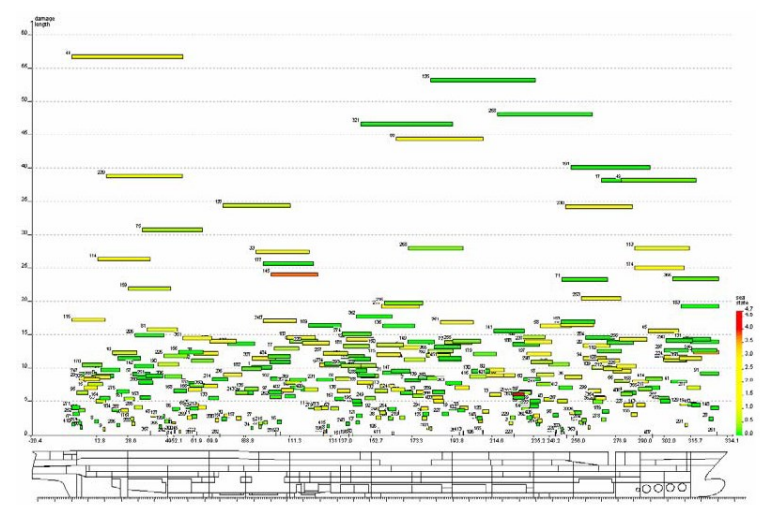

Figure 8: Monte Carlo Simulation Set up Collision (342 scenarios) - Large Cruise Ship

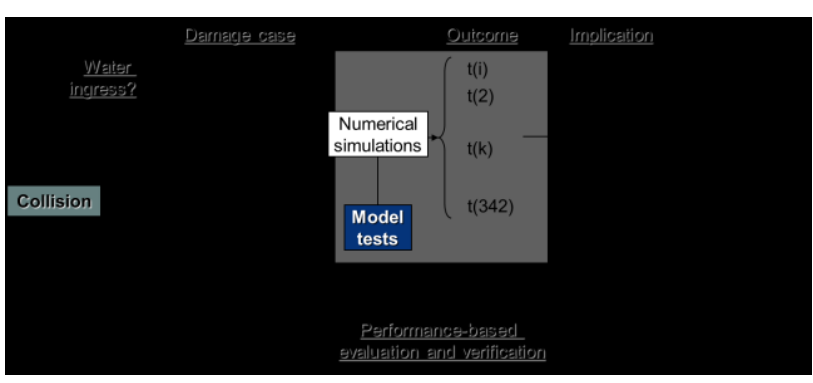

Figure 9: Monte Carlo Simulation and postprocessing set up - Collision (342 scenarios) Large Cruise Ship

Typical results are shown in Figures 10 and 11 for a RoPax and a Cruise Ship respectively as cumulative distribution functions of time to capsize. From the latter it will be seen that differences between the two methods of nearly an order of magnitude have been encountered and this led to renewed scrutiny of the probabilistic rules, as reported in (Vassalos and Jasionowski, 2007) that led to the EC-funded Project GOALDS. 


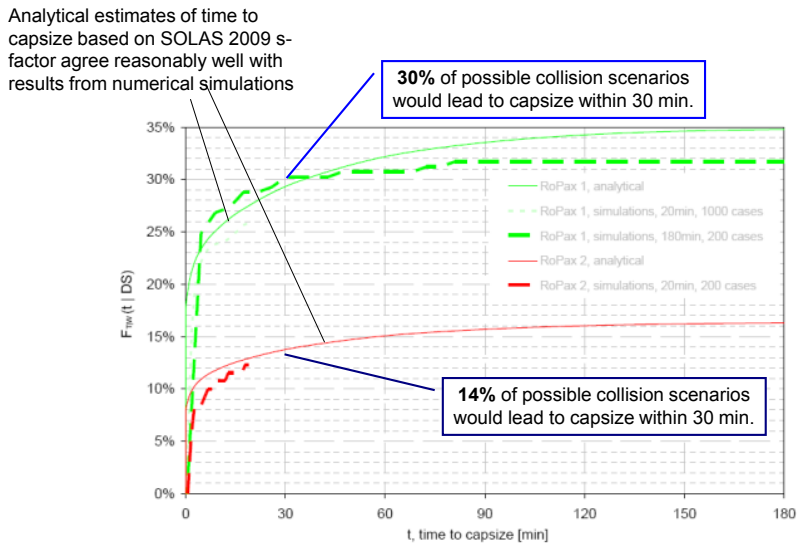

Figure 10: Probability Distributions of Time to Capsize (RoPax) - SOLAS 2009 Vs Direct Approach

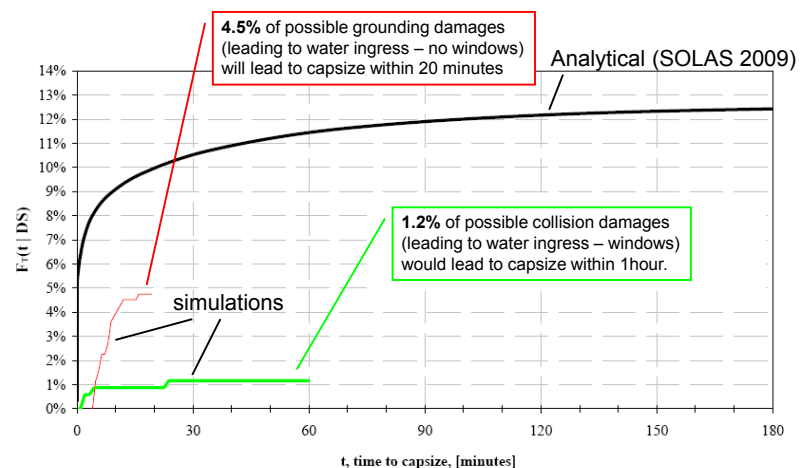

Figure 11: Probability Distributions of Time to Capsize (Cruise Ship) - SOLAS 2009 Vs Direct Approach

The results shown in the above figures offer another piece of evidence that the s-factor in current SOLAS does not represent the survivability of cruise ships by far. The fact that time to capsize for cruise ships is considerably longer than RoPax or indeed cargo ships on which the current SOLAS is based (i.e., half an hour) appears to have much larger impact on the ability to predict survivability of cruise ships than initially envisaged. Efforts to rectify this in Project GOALDS by encompassing cruise ship characteristics in the final formulation appear to have improved this situation as shown in Figure 12, in this case with an uncertainty band for $99.5 \%$ probability (Qi Chen, 2013). However, the fact that only one data point related to a cruise ship was used in such derivation has not had as full an impact on the final formulation of the s-factor as focusing on cruise ships alone would bring. Some new evidence based on the recently completed EMSA III project offers strong support in this respect.

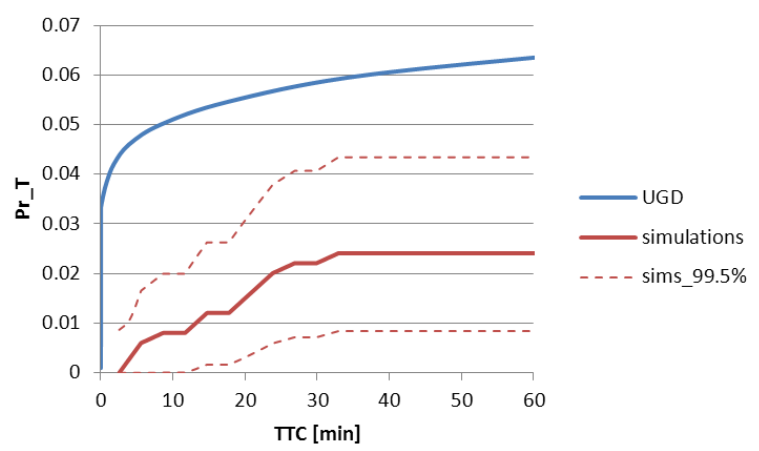

Figure 12: Probability Distributions of Time to Capsize (Cruise Ship) - GOALDS Project sfactor Vs Direct Approach

\section{RECOMMENDATIONS FOR A WAY FORWARD}

All the evidence available to date strongly suggests that the current SOLAS misrepresents the survivability of cruise ships. Continuing to group these with RoPax is no longer workable and more importantly largely unjustifiable. It is time to address survivability of cruise ships as a separate group of ships from RoPax. This will incentivise research to focus on these ships for the first time ever with the view to understanding the underlying characteristics that define survivability of cruise ships and to attempt to capture these in formulating and proposing a new s-factor for cruise ships. Following verification, application and calibration by the industry, this will lead to a legislative instrument, specifically for cruise ships, that will incentivise industry to seek continuous improvement and to facilitate designers in this quest. This time, it has to be the industry that takes initiative and leadership to put together a Joint Industry Project to target and accomplish this in a relatively short time. This is the only way forward! 


\section{CONCLUDING REMARKS}

- The general formulation of the s-factor for cargo ships was adopted as the harmonised solution for both cargo and passenger ships. This is irrational considering that cruise ships are vastly different to both types of ships on which the formulation is based.

- SOLAS 2009 formulation considerably underestimates cruise ship survivability. This implies that due credit is not given to the damage resilience of cruise ships, which, in turn, affects industry image (ships being seen less safe than they actually are).

- SOLAS 2009 formulation does not support best-practice design, meaning that potential solutions for improving cruise ship survivability will not be properly rated and hence dismissed. Adding to this is the risk of alienating the designers in that what they know to improve survivability in their designs does not appear to be justifiable.

- Emphasis on continuous safety improvement is, as a result, being hindered and safety culture undermined.

\section{REFERENCES}

Project HARDER, "Harmonisation of Rules and Design Rationale - HARDER", 2003, U Contact No. GDRB-CT-1998-00028, Final Technical Report.

Vassalos, D, Jasionowski, A and Guarin, L: "Passenger Ships Safety - Science Paving the Way", 2006. Journal of Marine Science and Technology, Vol. 2, Nos. 1-2, pp 63-71.

Svensen, T. and Vassalos, D: "Safety of Passenger/RoRo Vessels: Lessons Learnt from the North West European R\&D Project", Journal of Marine Technology,
Vol. 35, No. 4, pp191-200, October 1998.

EMSA III Project: "Combined Assessment of Cost-Effectiveness of Previous Parts, FSA Compilation and Recommendations for Decision Making”, 2016, European Maritime Safety Agency Report No.: 20150404, Rev. 3, Document No.: 18KJ9LI-60.

Lutzen, M, 2006, "Damage Distributions, Report No.: 2-22-D-2001-01-1, Task 2.2, EU Project No: GRD1- Harder", November 2001, 66 pp.; also in: Ship collision damage, $\mathrm{PhD}$ thesis, Department of Mechanical Engineering, Technical University of Denmark, Lyngby.

Jasionowski, A. and Vassalos, D., 2006, "Conceptualising Risk", STAB 2006, Rio de Janeiro, Brazil

Dogliani, M, Vassalos, D and Strang, T, 2004, "Evacuation Notation - A New Concept to Boost Passenger Evacuation Effectiveness in the Cruise Industry", COMPIT 2004, 3rd Int. Euro-Conference on Computer Applications and Information Technology in the Marine Industries, Parador Siguenza, Spain

Wendel, K, 1968, "Subdivision of Ships", Diamond Jubilee International Meeting, New York, June 1968, pp 12-1 to 12-21.

Tsakalakis, N., Cichowicz, J., Vassalos, D., 2010, "The concept of the capsize band revisited", 11th International Workshop on Stability of Ships and Ocean Vehicles, Wageningen

Jasionowski, A, Dodworth, K, Vassalos, D, 1999, "Proposal for Passenger SurvivalBased Criteria for Ro-Ro Vessels", International Shipbuilding Progress, Vol. 46, No 448

Cichowicz, J, Tsakalakis, N, Vassalos, D and Jasionowski, A, 2011, "Survivability of Passenger Vessels - Re-engineering of the s- 
Factor", Proceeding of the 12th International Ship Stability Workshop, Washington D.C., USA.

Tsakalakis, N:, 2012, "Performance-based Damage Survivability of Ships and Design Implications", PhD Thesis, University of Strathclyde, The Ship Stability Research Centre, Department of Naval Architecture, Ocean and Marine Engineering.

Vassalos, D, Jasionowski, A and Guarin, L, 2008, "Risk-Based Design: A Bridge too far?", OC 2008 Seakeeping and Stability, Osaka, Japan.

Jasionowski A, Vassalos D, Guarin L, 2004, "Theoretical Developments on Survival Time Post-Damage", Proceedings of the 7th Int Ship Stability Workshop, Shanghai.

Jasionowski A, Bulian G, Vassalos D, Francescutto A, Pawlowski, M, Maccari A, 2007, "Modelling survivability", SAFEDOR, D2.1.3, www.safedor.org.

Jasionowski, A, 2005: "An integrated approach to damage ship survivability assessment", $\mathrm{PhD}$, University of Strathclyde.

Vassalos, D and Jasionowski, A, 2007, "SOLAS 2009 - Raising the Alarm", 9 th $^{\text {th }}$ International Stability Workshop, Hamburg, Germany

Papanikolaou, A, Hamman, R, Lee, BS, Lemoine, L, Mains, C, Olufsen O,, Tvedt, E, Vassalos, D and Zaraphonitis, G:, 2013, "GOALDS: Goal-Based Damage Stability of Passenger Ships", Transactions SNAME, Captain Joseph H. Linnard Prize.

Chen, Q, 2013 "Uncertainty Quantification in Assessment of Damage Ship Survivability" PhD Thesis, Strathclyde. 